\title{
Early diagnosis of acute myocardial infarction by a newly developed rapid immunoturbidimetric assay for myoglobin
}

Johannes Mair, Erika Artner-Dworzak, Peter Lechleitner, Bernhard Morass, Jörn Smidt, Ina Wagner, Franz Dienstl, Bernd Puschendorf

Abstract

Objective-To evaluate a rapid immunoturbidimetric assay for myoglobin and to investigate its clinical usefulness in the early detection of acute myocardial infarction.

Design-Prospective study. Immunoturbidimetrically determined myoglobin concentrations were compared with radioimmunoassay results obtained with the same blood samples. The diagnostic performance of myoglobin determination was compared with creatine kinase and creatine kinase $M B$ activity (current standard of routine diagnosis).

Settings-Part 1: coronary care unit. Part 2: emergency room in a university hospital.

Patients-Part 1: 30 patients with acute myocardial infarction admitted not later than four hours (median two hours) after the onset of symptoms. Part 2: 126 patients admitted to the emergency room with chest pain not caused by trauma ( 51 cases of acute myocardial infarction, 51 cases of angina pectoris, and 24 cases of chest pain not related to coronary artery disease).

Interventions-Part 1: routine treatment including intravenous thrombolytic treatment (28 patients). Part 2: routine emergency treatment without thrombolytic treatment.

Main outcome measures-The analytical quality of the immunoturbidimetric myoglobin assay and a comparison between the myoglobin assay and creatine kinase and creatine kinase $M B$ for diagnostic sensitivity and performance.

Results-The immunoturbidimetric myoglobin assay was fast and convenient and gave myoglobin determinations of high analytical quality. The concentration of myoglobin increased, peaked, and returned to the reference range significantly earlier than creatine kinase (p $\leqslant 0.0001)$ and creatine kinase MB ( $p \leqslant$ 0.0002). Before thrombolytic therapy was started the diagnostic sensitivity of myoglobin was significantly higher than that of creatine kinase $M B$ activity $0-6 \mathrm{~h}$ after the onset of chest pain and significantly higher $\left(\begin{array}{llll}0.82 & v & 0.29) & \text { than }\end{array}\right.$ creatine kinase $2-4 \mathrm{~h}$ after the onset of chest pain. In almost all patients $(92 \%)$ plasma myoglobin concentrations were increased 4-6 h after the onset of chest pain.

Conclusion-Myoglobin was more sensitive in detecting early myocardial infarction than creatine kinase and creatine kinase MB activity. Immunoturbidimetric myoglobin measurements could be useful in the early evaluation of patients with suspected myocardial infarction because this assay takes less than two minutes.

\section{(Br Heart J 1992;68:462-8)}

Apart from the patient who presents with classic symptoms and typical electrocardiographic changes, the early detection of acute myocardial infarction based on conventional clinical and electrocardiographic findings alone is difficult. ${ }^{1}$ Most patients with acute myocardial infarction show some electrocardiographic changes, but these changes are diagnostic in only about one third of patients with acute myocardial infarction when they present at the emergency room. ${ }^{2-4}$ The use of thrombolytic therapy for myocardial salvage reinforces the need for early and rapid identification and confirmation of acute myocardial infarction within four to six hours after the onset of the infarct-related symptoms. ${ }^{5}$ Creatine kinase and creatine kinase $\mathrm{MB}$ activity, which are the most frequently assayed biochemical markers in emergency laboratories, do not rise in serum until the fourth to eighth hour after the onset of chest pain, ${ }^{6}$ and so are not sensitive enough for diagnosis during the early stages of acute myocardial infarction. Myoglobin, by contrast, is an early sensitive marker of myocardial cell injury. ${ }^{7-10}$ Until recently radioimmunoassay and latex agglutination were the two techniques available for myoglobin determination. Radioimmunoassays are time consuming and therefore not suited for use in emergency room laboratories. Latex agglutination is a rapid test but the results are only semi-quantitative estimations of serum myoglobin concentrations. Under optimal laboratory conditions, the latex test is a reliable method of estimating myoglobin concentrations. Performed occasionally by a technical assistant on duty or when used as a bedside test, however, its results correlated rather poorly with the radio-

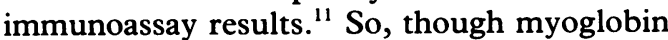
is known to be an early sensitive indicator of acute myocardial infarction and to be a reliable
Fritz-Preglstrasse 3, A-6020 Innsbruck, Austria.

Accepted for publication 11 May 1992 
non-invasive predictor of the outcome of therapeutic reperfusion, ${ }^{1213}$ it has not been widely used in the early evaluation of patients with suspected acute myocardial infarction.

We have evaluated a rapid, quantitative immunoturbidimetric myoglobin assay in the early detection of acute myocardial infarction.

\section{Patients and methods}

PATIENTS

The study was carried out in two parts.

Part 1

This part of our study was performed to confirm the differences in the time courses of myoglobin, creatine kinase $M B$, and total creatine kinase activity in plasma after acute myocardial infarction and to establish a correlation between myoglobin concentrations measured by radioimmunoassay and by immunoturbidimetry. Thirty consecutive patients with subsequently confirmed myocardial infarction ( 22 men and eight women) aged 40-83 years (median 61 years) presented to the hospital coronary care unit within four hours after the onset of symptoms and gave informed consent for extra blood samples to be drawn. Patients presenting to the coronary care unit more than four hours after the onset of symptoms were excluded, because at this stage of acute myocardial infarction there is a high probability of increased creatine kinase and creatine kinase $\mathrm{MB}$ activity and an increased myoglobin plasma concentration. The median time to admission was two hours (range 30-240 minutes). All patients subsequently proved to have sustained an acute myocardial infarction (27 $Q$ wave and three non- $Q$ wave myocardial infarctions: 12 anterior and 18 inferior wall infarctions). Myocardial infarction was diagnosed independently by two cardiologists according to the World Health Organisation criteria ${ }^{14}$ based on the patient's clinical history and symptoms, electrocardiographic abnormalities, and serum enzyme findings and without knowledge of the myoglobin results. Treatment was determined by the clinical circumstances. Fifteen patients were given intravenous streptokinase ( 1.5 million units in 60 minutes), two patients were given intravenous urokinase ( 2 million units in 10 to 15 minutes), and 11 patients were given intravenous alteplase (initial bolus of $10 \mathrm{mg}$, followed by $50 \mathrm{mg}$ during the first hour and $20 \mathrm{mg}$ each during the second and third hour after admission). Two patients were not given thrombolytic treatment because of contraindications. All patients additionally received routine coronary care and were treated with intravenous heparin, aspirin, nitrates, occasionally $\beta$ blockers, and antiarrhythmic drugs as needed. Peripheal venous blood samples were collected from an indwelling forearm catheter or by venepuncture before treatment in the coronary care unit. Samples were collected hourly for the first 10 hours, then at 12,16 , $20,24,32,40,48$ hours after admission, and thereafter daily until biochemical markers returned to normal. Patient care requirements occasionally prevented a sample being taken.
On the basis of recent findings, ${ }^{15}$ coronary angiography was performed only selectively usually several days after admission. So the occurrence of reperfusion in $Q$ wave infarction could not be observed directly by coronary angiography but had to be inferred from less definitive non-invasive criteria. It is well established, both clinically ${ }^{1216-18}$ and experimentally, ${ }^{1920}$ that reperfusion in $\mathrm{Q}$ wave infarction results in a more rapid increase in creatine kinase and myoglobin and in early peaks of both proteins. Early reperfusion was assumed to have occurred ( $95 \%$ probability of correct classification) if there was both a myoglobin peak within seven hours and a creatine kinase peak within 16 hours after the onset of chest pain. ${ }^{13}$ Eighteen of 25 patients with $Q$ wave myocardial infarction given thrombolytic therapy were classified as "reperfused early".

Part 2

In the second part of the study we evaluated the immunoturbidimetric myoglobin determination as a rapid screening assay for myocardial infarction in patients with chest pain presenting to the emergency room. One hundred and twenty six consecutive patients in the hospital's department of internal medicine with chest pain as their major symptom were investigated. The study population did not include patients with chest contusion or injured patients. Fifty one patients subsequently proved to have sustained an acute myocardial infarction (median delay from the onset of chest pain to admission $2.25 \mathrm{~h}$, range $0.5-14.5 \mathrm{~h}$ ), which was diagnosed by two cardiologists according to the WHO criteria given earlier. Twenty six patients sustained a $Q$ wave acute myocardial infarction (median delay $1.75 \mathrm{~h}$, interquartile range $1-3.5$ h) and 25 a non-Q wave acute myocardial infarction (median delay $4 \mathrm{~h}$, interquartile range $1-7 \cdot 15$ hours). Fifty one patients presented with angina pectoris. A further 24 patients had chest pain not related to coronary artery disease (three cases of supraventricular tachycardia, four pneumonia with pleurisy, one obstructive pulmonary disease, four musculoskeletal disorders, three pericarditis, six pulmonary embolism, two oesophagitis, and one case of hypertensive crisis). In these patients a single blood sample was drawn immediately after presentation to the emergency room. All patients received routine emergency treatment. All patients with acute myocardial infarction were transferred to the coronary care unit before the start of thrombolytic therapy. None of these patients with acute myocardial infarction had been given thrombolytic agents before admission to the hospital.

\section{LABORATORY ANALYSIS \\ Blood collection}

Blood was collected in tubes coated with EDTA. Creatine kinase and creatine kinase MB activities were assayed immediately after collection. Blood samples for the measurement of myoglobin concentrations were centrifuged immediately and the plasma subsequently 
frozen and stored at $-20^{\circ} \mathrm{C}$ until determination. Under these conditions myoglobin could be assayed within at least four weeks (maximum period of storage) after collection without any decline in concentration.

Creatine kinase and creatine kinase $M B$ activity Total creatine kinase and creatine kinase $M B$ activities were measured $\left(25^{\circ} \mathrm{C}\right)$ with $\mathrm{N}$-acetylcysteine activated, optimised ultraviolet test kits obtained from Merck (Darmstadt, Germany). Creatine kinase MB activity was measured by means of immunoinhibition based on the presence of inhibiting creatine kinase $M$ antibodies. According to the manufacturer's recommendations, a total creatine kinase activity of $70 \mathrm{U} / 1$ for women and $80 \mathrm{U} / 1$ for men was used as the upper limit of the reference interval. For creatine kinase MB activity the reference range goes up to $10 \mathrm{U} / 1$. Creatine kinase $\mathrm{MB}$ activities of $>10 \mathrm{U} / 1$ and an increase of $>6 \%$ in total creatine kinase activity were assumed to indicate myocardial muscle cell damage.

\section{Myoglobin}

Myoglobin radioimmunoassay-Myogiobin was determined by a commercially available radioimmunoassay (Byk-Sangtec, Dietzenbach, Germany). The manufacturer's upper limit of the reference interval is $80 \mu \mathrm{g} / 1$.

Immunoturbidimetric myoglobin assay-A myoglobin assay (Turbiquant myoglobin, Behringwerke AG, Marburg, Germany) for use with the Behring Turbitimer analyser was used for rapid immunoturbidimetric determination of myoglobin concentrations in plasma. ${ }^{21}$ This assay is based on polystyrene particles coated with rabbit anti-human myoglobin antibodies. In an immunochemical reaction these particles form agglutinates with the myoglobin contained in serum or plasma. The increase in turbidity is measured photometrically. ${ }^{22}$ Quantitative results are available about a minute after the start of the assay. The detection limit of the assay is $50 \mu \mathrm{g} / 1$ and the measurement range 50-650 $\mu \mathrm{g} / \mathrm{l}$. The concentrations of myoglobin in EDTA plasma from 100 apparently healthy subjects (blood donors with no history of cardiovascular diseases) (41 men and 59 women aged 19-65 (median 44 years)) were measured by this new method to obtain a reference interval calculated by nonparametric determination of percentiles.

DATA ANALYSIS

Median, interquartile range, and percentiles were calculated to describe continuous variables. The association between continuous variables was analysed by the Spearman rank correlation test. The Wilcoxon signed rank test and $\chi^{2}$ test (or Fisher's exact test where appropriate) were used for between group comparisons. Confidence intervals were calculated according to the method of Gardner and Altman. ${ }^{23} \mathrm{~A} \mathrm{p}$ value of $<0.05$ was regarded as significant. Bonferroni adjustment was used for comparison of more than two groups. Sensitivity, specificity, efficiency, positive and negative predictive values, likelihood ratio, and Youden index (sensitivity + specificity -1 ) were calculated to describe the performance of myoglobin, creatine kinase, and creatine kinase $\mathrm{MB}$ activity in the early diagnosis of myocardial infarction in patients in the emergency room. ${ }^{24-26}$

\section{Results}

IMMUNOTURBIDIMETRIC ASSAY FOR MYOGLOBIN Reference interval of myoglobin concentrations in plasma by the immunoturbidimetric assay

Figure 1 shows the distribution of myoglobin concentrations in 100 healthy individuals. In most the myoglobin concentration was below the detection limit of the assay. The upper limit of the reference interval (cut off value), calculated as the $97.5 \%$ percentile, was $70 \mu \mathrm{g} / \mathrm{l}$. The age or sex of the reference subjects did not influence the myoglobin concentrations. The correlation coefficient between myoglobin concentrations and age was 0.08 . There was no significant $(p=0 \cdot 27)$ difference between myoglobin concentrations in men and women.

\section{Correlation with radioimmunoassay method}

Myoglobin was simultaneously determined by both methods in serial blood samples taken from 30 patients with acute myocardial infarction. There was a good correlation between the immunoturbidimetric assay and radioimmunoassay for myoglobin time courses of individual patients with myocardial infarction. The median correlation coefficient was 0.96 (interquartile range 0.91-0.97). However, values obtained by the immunoturbidimetric assay were usually lower than those obtained by radioimmunoassay.

\section{Interference}

Rheumatoid factors (tested for a concentrations up to $1260 \mathrm{IU} / \mathrm{ml}$ ) did not interfere with the assay. The myoglobin assay is usually not disturbed by icteric, haemolytic, and lipaemic samples. However, if the absorbance of the sample is too high before the reaction starts (for example, because of strong absorption caused by haemolysis or hypertriglyceridaemia), the analyser generates a warning message and the assay cannot be done. Such samples can be

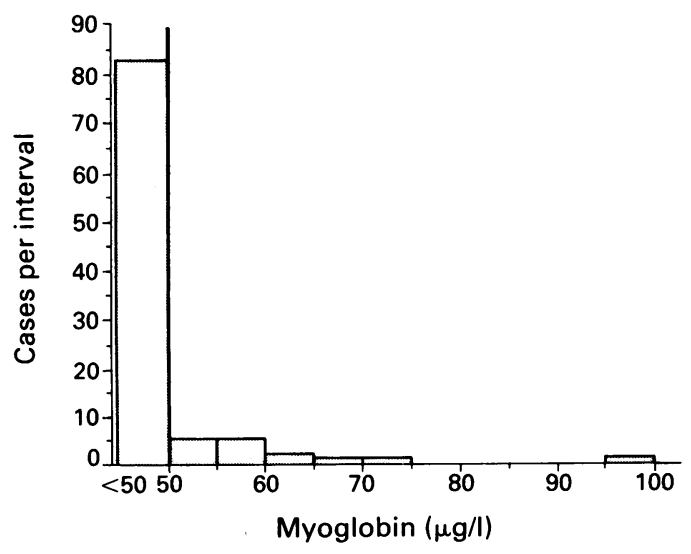

Figure 1 Plasma concentrations of myoglobin in 100 healthy volunteers. The bars represent the number of cases including the lowest value of each interval. The detection limit of the assay is $50 \mu \mathrm{g} / \mathrm{l}$ (bold line). 
retested after manual predilution. Hypertriglyceridaemic samples can be also clarified by centrifugation (10 $\mathrm{min}$ at approximately $15000 \mathrm{~g}$ ) and retested.

\section{Reproducibility}

The calculation of the coefficients of variation for patients' and control samples was based on 20 measurements each. The intraassay coefficients of variation ranged from $2 \cdot 2 \%$ to $6.8 \%$ and the interassay coefficients of variation from $5.9 \%$ to $11 \cdot 7 \%$. The highest coefficients of variation were found for samples with low myoglobin concentrations.

\section{TIME COURSES OF MYOGLOBIN, CREATINE} KINASE, AND CREATINE KINASE MB ACTIVITY IN MYOCARDIAL INFARCTION

Figure 2 summarises the time courses of myoglobin concentrations of 30 patients with myocardial infarction. Thrombolytic therapy resulted in early reperfusion in 18 of 25 patients with $Q$ wave infarction. The time courses of myoglobin, creatine kinase, and creatine kinase $M B$ activities in patients with non- $Q$ wave myocardial infarctions resembled those of patients with $Q$ wave myocardial infarctions with early reperfusion. Table 1 shows the criteria describing the release kinetics of myoglobin, creatine kinase, and creatine kinase MB activities. An abnormal increase, the peak concentration, and return into the reference range occurred significantly earlier for myoglobin than for creatine kinase ( $p \leqslant$ $0.0001)$ and creatine kinase $M B$ activity ( $p \leqslant$ $0 \cdot 0002$ ). The median difference between the first appearances of myoglobin and creatine kinase and the first appearances of myoglobin and creatine kinase MB was one hour each (95\% CI 1-2 h). In these patients the diagnostic sensitivity of myoglobin was significantly ( $p$ $<0.05$ ) higher than that of both creatine kinase and creatine kinase $\mathrm{MB}$ activity at 3.5 and four hours after the onset of symptoms (fig 3 ). The diagnostic sensitivity of myoglobin was 0.5 $(50 \%) 3.4$ hours after the onset of symptomsa value that creatine kinase activity only reached after 4.8 hours and creatine kinase $M B$ after five hours respectively (table 1). All patients had increased myoglobin concentrations (100\% sensitivity) at six hours and increased creatine kinase and creatine kinase $\mathrm{MB}$ activities at 12 hours after the onset of chest pain (fig 3 ). The median peak values of myoglobin, creatine kinase, and creatine kinase MB were attained early after admission because of the high percentage $(72 \%)$ of patients with $Q$ wave myocardial infarction who achieved early reperfusion. The magnitude of increase (calculated as the peak value divided by the cut off value) of myoglobin was significantly (table 1) greater than for creatine kinase activity ( $p=0.0007)$ and creatine kinase MB activity (0.0001). The period when plasma concentrations of myoglobin were increased after acute myocardial infarction that allowed a diagnosis based on increased biochemical markers was significantly shorter than that of creatine kinase $(p=0.0001)$ and creatine kinase MB activity ( $p=0.0002)$. The correla-

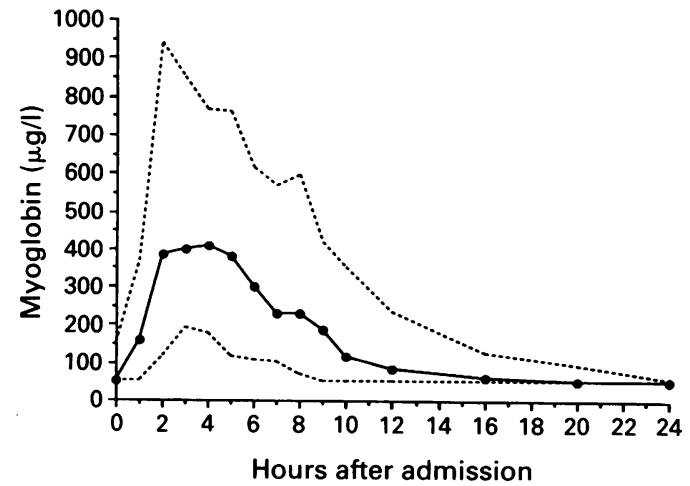

Figure 2 Time course of plasma concentrations of myoglobin in 30 patients with acute myocardial infarction (27 $Q$ wave, three non- $Q$ wave) presenting within four hours after the onset of symptoms. Data are given as median (solid line) and interquartile ranges (broken lines). Twenty five patients with $Q$ wave acute

myocardial infarctions were treated with intravenous thrombolytic agents, resulting in early reperfusion in 18.

tion coefficient between peak values of myoglobin and creatine kinase was 0.69 ( $p=$ 0.0002 ) and between myoglobin and creatine kinase $M B$ it was $0.68(\mathrm{p}=0.0002)$.

DIAGNOSTIC PERFORMANCE OF MYOGLOBIN, CREATINE KINASE, AND CREATINE KINASE MB ACTIVITY WHEN USED TO DETECT ACUTE MYOCARDIAL INFARCTION IN EMERGENCY ROOM PATIENTS WITH CHEST PAIN

In this group the prevalence of myocardial infarction was 0.4 (pretest odds 0.68 ). The median creatine kinase activity in patients with acute myocardial infarction $(n=51)$ was $68 \mathrm{U} / 1$ (interquartile range $47-142 \mathrm{U} / 1$ ), the median creatine kinase $\mathrm{MB}$ activity was $10 \mathrm{U} / 1$ (interquartile range 4-12 U/1). In these patients myoglobin concentrations ranged from $<50$ $\mu \mathrm{g} / 1$ to $2990 \mu \mathrm{g} / 1$. Eighteen patients with acute myocardial infarction had myoglobin concentrations below the detection limit $(50 \mu \mathrm{g} / \mathrm{l})$ of the immunoturbimetric assay. The median creatine kinase activity in patients with angina pectoris was $37 \mathrm{U} / 1$ (interquartile range 26-68 $\mathrm{U} / \mathrm{l})$, the median creatine kinase $\mathrm{MB}$ activity was $4 \mathrm{U} / 1$ (interquartile range $4-6 \mathrm{U} / \mathrm{l}$ ). In this group $(n=51)$ myoglobin concentrations ranged from $<50 \mu \mathrm{g} / 1$ to $72.6 \mu \mathrm{g} / 1$ and were $<50 \mu \mathrm{g} / 1$ in 43 cases. In patients with chest

Table 1 Release kinetics of myoglobin, creatine kinase (CK), and creatine kinase $M B$ activity in 30 patients with acute myocardial infarction (median (95\% CI))

\begin{tabular}{llll}
\hline & $\begin{array}{l}C K M B \\
\text { activity }\end{array}$ & $\begin{array}{l}\text { CK } \\
\text { activity }\end{array}$ & Myoglobin \\
\hline Cut off value & $10 \mathrm{U} / 1$ & $70 \mathrm{U} / 1$ & $70 \mu \mathrm{g} / 1$ \\
Time to first increased & 5 & $4 \cdot 8$ & $3 \cdot 4$ \\
plasma values (h) & $(4-6)$ & $(3 \cdot 5-5 \cdot 3)$ & $(2 \cdot 8-4 \cdot 0)$ \\
Time to peak (h) & $11 \cdot 3$ & 13 & $5 \cdot 9$ \\
& $(11-14 \cdot 3)$ & $(11 \cdot 5-15 \cdot 8)$ & $(4 \cdot 5-7 \cdot 5)$ \\
& 53 & 74 & $21 \cdot 5$ \\
Return to normal (h) & $(36 \cdot 3-76)$ & $(50-96)$ & $(17 \cdot 8-26 \cdot 0)$ \\
& 50 & 68 & 17 \\
Period of increased & $(32 \cdot 3-71 \cdot 8)(46-78)$ & $(12 \cdot 8-23 \cdot 0)$ \\
plasma values (h) & $4 \cdot 5$ & 7 & $9 \cdot 3$ \\
Magnitude of increase & $(3 \cdot 5-7 \cdot 7)$ & $(4 \cdot 3-12)$ & $(5 \cdot 5-23 \cdot 6)$ \\
& & & \\
\hline
\end{tabular}

The onset of symptoms (chest pain) was the reference point for the calculation of first appearance in blood, time to peak value, and time of return to normal.

and time of return to normal.
$\star$ Peak value divided by cut off valuc. Twenty five patients with * Peak value divided by cut off valuc. Twenty five patients with
$Q$ wave acute myocardial infarctions were given intravenous $Q$ wave acute myocardial infarctions were given intravenous
thrombolytic treatment, which resulted in early reperfusion in 18 . 


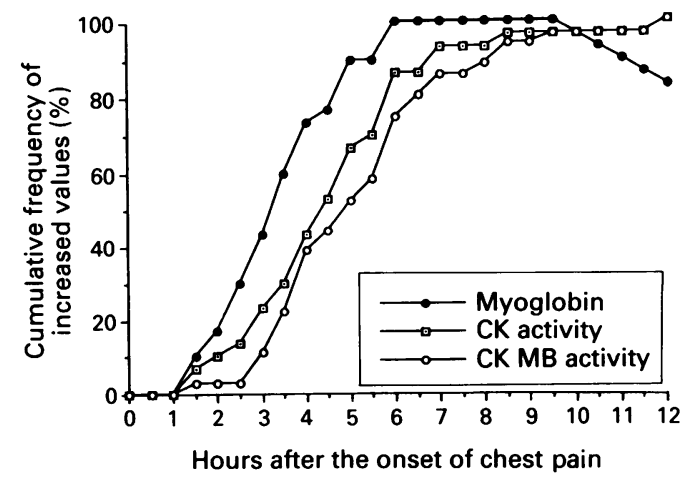

Figure 3 Diagnostic sensitivity of myoglobin, creatine kinase (CK), and creatine kinase $M B$ activity during the first hours after the onset of symptoms (with patients receiving thrombolytic treatment included) in the 30 patients shown in fig 2. Symbols represent the number of increased values (\%) including the lowest value of each time classification ( 30 minute intervals).

pain not related to coronary artery disease the median creatine kinase activity was $33 \mathrm{U} / 1$ (interquartile range 15-59 $\mathrm{U} / \mathrm{l}$ ) and the median creatine kinase $\mathrm{MB}$ activity was $4 \mathrm{U} / 1$ (interquartile range 4-5 U/1). Myoglobin concentrations measured in the same blood samples $(\mathrm{n}=24)$ ranged from $<50 \mu \mathrm{g} / 1$ to $162 \mu \mathrm{g} / 1$ and were $<50 \mu \mathrm{g} / 1$ in 23 patients. Figure 4 shows the influence of the cut off value on the sensitivity, specificity, and Youden index of myoglobin in the discrimination between acute myocardial infarction and non-acute myocardial infarction in patients presenting to the emergency room with symptoms that suggested myocardial infarction. The myoglobin cut off value that yielded the highest Youden index lay somewhere below the detection limit of the immunoturbidimetric assay. The myoglobin cut off value of $70 \mu \mathrm{g} / \mathrm{l}$ (established in healthy blood donors) gave good sensitivity $(0.45)$ and specificity (0.91). Table 2 shows various indices of performance of the diagnostic tests. For the calculations presented in table 2 we used either the cut off values recommended by the manufacturer (creatine kinase and creatine kinase MB activity) or our own cut off values established in healthy blood donors (myoglobin). Twenty two (43\%) of the patients with acute myocardial infarction were admitted to the hospital either within two hours or more than six hours after the onset of chest pain.



Figure 4 Diagnostic performance when different cut off plasma concentrations of myoglobin were used to detect acute myocardial infarction in 126 patients with chest pain admitted to the emergency room. The prevalence of acute myocardial infarction was 0.4 .
Owing to this distribution of delay from the onset of pain to admission only the difference between the sensitivities of myoglobin and creatine kinase $M B$ was statistically significant when all patients were included in the calculation of the sensitivity. Subgroup analysis of the sensitivities of myoglobin and creatine kinase in patients admitted during the $2-4$ hour period (limits included), however, also showed a significantly ( $p=0.016)$ higher sensitivity for myoglobin compared with creatine kinase $(0.76$ $v 0 \cdot 29)$. Table 3 shows the false positive diagnoses of acute myocardial infarction for creatine kinase, creatine kinase MB activity, or the immunoturbidimetric myoglobin assay. The specificity, positive predictive value, and likelihood ratio of myoglobin were significantly higher than for creatine kinase. The sensitivity and Youden index for myoglobin were significantly higher than for creatine kinase $M B$ activity (table 2 ).

Fifteen patients with myocardial infarction presented with a non-diagnostic electrocardiogram to the emergency ward within six hours (median two hours) of the onset of chest pain. In these potential candidates for fibrinolytic therapy the sensitivity of myoglobin $(0 \cdot 6$, $95 \%$ CI 0.35 to 0.85 ) was significantly higher than that of creatine kinase $(0.33,95 \%$ CI 0.09 to 0.57$)$ and creatine kinase $\mathrm{MB}$ activity $(0.13$, $95 \% \mathrm{CI}$ interval 0 to $0 \cdot 3$ ).

\section{MYOGLOBIN, CREATINE KINASE, AND CREATINE} KINASE MB ACTIVITY SENSITIVITIES BEFORE THE START OF THROMBOLYTIC THERAPY

Table 4 shows the sensitivities of myoglobin, creatine kinase, and creatine kinase $\mathrm{MB}$ activity during the early stages of acute myocardial infarction before the start of thrombolytic treatment. This table is based on the combined analysis of the data from emergency room patients and from the blood samples taken from patients with myocardial infarction in part 1 of our study at admission to the coronary care unit before thrombolytic treatment. Patients who had had cardiopulmonary resuscitation or defibrillation therapy were also excluded from data analysis. Myoglobin was significantly more sensitive than creatine kinase MB during the $0-6$ hour period and significantly more sensitive than creatine kinase activity during the 2-4 hour period after the onset of chest pain. Almost all patients $(92 \%)$ had increased plasma myoglobin concentrations during the 4-6 hour period.

\section{Discussion}

The immunoturbidimetric myoglobin assay allows fast and convenient myoglobin determinations of high analytical quality. Intra and inter assay coefficients of variation are within acceptable limits. Interference by rheumatoid factors, haemoglobin, bilirubin, and triglycerides was negligible under routine conditions. The correlation between immunoturbidimetric results and radioimmunoassay myoglobin results was good. The results of immunoturbidimetric myoglobin assay are available within 1-2 minutes of the start of the assay. These properties make this method 
Table 2 Comparison of myoglobin, creatine kinase (CK) and creatine kinase $M B$ activity in the emergency room diagnosis on admission of acute myocardial infarction in 126 patients with chest pain

\begin{tabular}{llll}
\hline & $C K M B$ & $C K$ & \\
& activity & activity & Myoglobin \\
\hline Cut off value & $10 \mathrm{U} / 1$ & $70 \mathrm{U} / 1$ & $70 \mu \mathrm{g} / \mathrm{l}$ \\
Sensitivity & 0.32 & 0.44 & 0.45 \\
Specificity & $(0.24$ to 0.4$)$ & $(0.35$ to 0.53$)$ & $(0.36$ to 0.54$)$ \\
& 0.92 & 0.82 & 0.91 \\
Positive predictive value & $(0.87$ to 0.97$)$ & $(0.75$ to 0.89$)$ & $(0.86$ to 0.96$)$ \\
& 0.72 & 0.63 & 0.77 \\
Negative predictive value & $(0.53$ to 0.91$)$ & $(0.53$ to 0.71$)$ & $(0.70$ to 0.84$)$ \\
Efficiency & 0.66 & 0.68 & 0.71 \\
& $(0.58$ to 0.74$)$ & $(0.6$ to 0.76$)$ & $(0.63$ to 0.79$)$ \\
Youden index & 0.67 & 0.67 & 0.72 \\
& $(0.59$ to 0.75$)$ & $(0.59$ to 0.75$)$ & $(0.64$ to 0.80$)$ \\
Likelihood ratio & 0.24 & 0.26 & 0.36 \\
& $(0.17$ to 0.31$)$ & $(0.18$ to 0.34$)$ & $(0.28$ to 0.44$)$ \\
& 4 & 2.5 & 5 \\
& $(1.6$ to 6.4$)$ & $(1.8$ to 3.2$)$ & $(2.6$ to 7.4$)$ \\
\hline
\end{tabular}

The prevalence of acute myocardial infarction was 0.4 (pretest odds 0.68 ). The delay of myocardial infarction patients from the onset of symptoms to admission ranged from 0.5 to 14.5 hours (median $2 \cdot 25$ hours). $95 \%$ confidence intervals are given in parentheses.

Table 3 Patients with false positive myoglobin, creatine kinase (CK), or creatine kinase $M B$ test results

\begin{tabular}{|c|c|c|c|c|}
\hline Patient & Diagnosis & $\begin{array}{l}\text { CK activity } \\
\text { (cut off value } \\
70 \text { U/l) }\end{array}$ & $\begin{array}{l}\text { CK MB activity } \\
\text { (cut off value } \\
10 \mathrm{U} / \mathrm{l} \text { ) }\end{array}$ & $\begin{array}{l}\text { Myoglobin } \\
\text { (cut off value } \\
70 \mu g / l)\end{array}$ \\
\hline 1 & Angina pectoris & 87 & 13 & 65 \\
\hline 2 & Angina pectoris & 201 & 4 & $<50$ \\
\hline 3 & Musculoskeletal disorder & 77 & 4 & $<50$ \\
\hline 4 & Pulmonary embolism & 108 & 8 & $<50$ \\
\hline 5 & Angina pectoris & 94 & 4 & $<50$ \\
\hline 6 & DC countershock therapy & 123 & 13 & 244 \\
\hline 7 & CK increase, unknown origin & 138 & 4 & $<50$ \\
\hline 8 & Angina pectoris & 110 & 7 & $<50$ \\
\hline 9 & Angina pectoris & 170 & 7 & $54 \cdot 2$ \\
\hline 10 & Pericarditis & 146 & 4 & $<50$ \\
\hline 11 & Angina pectoris & 97 & 4 & $<50$ \\
\hline 12 & Pericarditis & 114 & 4 & $62 \cdot 8$ \\
\hline 13 & Seizure & 46 & 6 & 162 \\
\hline 14 & Angina pectoris & 114 & 5 & $72 \cdot 6$ \\
\hline 15 & Angina pectoris & 123 & 6 & $56 \cdot 2$ \\
\hline 16 & Gastritis & 87 & 4 & $<50$ \\
\hline
\end{tabular}

ideally suited for the emergency room laboratory.

More extended and frequent blood sampling than in earlier studies confirmed previous reports $^{7-10}$ and showed statistically significant and clinically relevant differences in the time courses of myoglobin concentration and creatine kinase and creatine kinase $\mathrm{MB}$ activity after myocardial infarction and treatment with thrombolytic agents. The same patients were tested for myoglobin, creatine kinase, and creatine kinase $\mathrm{MB}$, which cancelled out all possible biases that could have distorted the results (that is, delay between attack and treatment, different treatments, and size and site of infarction etc). Serial measurement of myoglobin in blood is a useful non-invasive means of monitoring the success of therapeutic recanalisation. ${ }^{1213}$ Reperfusion alters the release kinetics of myoglobin and other cardiac cytoplasmic proteins by washout from injured or dead myocytes. ${ }^{18-20} \mathrm{~A}$ rapid increase and subsequent decrease in cardiac cytoplasmic proteins occur as perfusion is restored to the ischaemic regions of the heart. Ellis et al proposed a $4 \cdot 6$-fold increase in myoglobin during the first two hours after the initial attempt to restore perfusion in the infarct related vessel compared with myoglobin concentrations at the outset to discriminate be-
Table 4 A comparison of the sensitivity of myoglobin and creatine kinase (CK), and creatine kinase $M B$ in the early stages of myocardial infarction (before thrombolytic treatment)

\begin{tabular}{|c|c|c|c|}
\hline $\begin{array}{l}\text { Delay between } \\
\text { onset of } \\
\text { symptoms and } \\
\text { blood sampling }\end{array}$ & Myoglobin & $C K$ & $\begin{array}{l}C K M B \\
\text { activity }\end{array}$ \\
\hline$\leqslant 2 \mathrm{~h}$ & $\begin{array}{l}0.23 \\
(0.1 \text { to } 0.35)\end{array}$ & $\begin{array}{l}0.13 \\
(0.02 \text { to } 0.23)\end{array}$ & $\begin{array}{l}0.05 \\
(0.0 \text { to } 0.12)\end{array}$ \\
\hline$>2$ and $\leqslant 4 h$ & $\begin{array}{l}0.82 \\
(0.69 \text { to } 0.95)\end{array}$ & $\begin{array}{l}0.29 \\
(0.14 \text { to } 0.44)\end{array}$ & $\begin{array}{l}0.23 \\
(0.08 \text { to } 0.38)\end{array}$ \\
\hline$>4$ and $\leqslant 6$ & $\begin{array}{l}0.92 \\
(0.77 \text { to } 1.0)\end{array}$ & $\begin{array}{l}0.75 \\
(0.51 \text { to } 0.99)\end{array}$ & $\begin{array}{l}0.33 \\
(0.14 \text { to } 0.61)\end{array}$ \\
\hline
\end{tabular}

Calculations of sensitivities are based on cut off values: $70 \mu \mathrm{g}$ (myoglobin), $70 \mathrm{U} / 1$ (creatine kinase activity), and $10 \mathrm{U} / \mathrm{l}$ (creatine kinase MB activity). $95 \%$ confidence intervals are given in parentheses. Blood samples obtained from patients after cardiopulmonary resuscitation, defibrillation, or thrombolytic therapy were excluded from data analysis.

tween myocardial infarction patients with successful and failed reperfusion after thrombolytic therapy. ${ }^{12}$ Rapid prediction of the outcome of therapeutic reperfusion by immunoturbidimetric myoglobin measurements could become increasingly important by allowing the use of alternative, invasive means of recanalisation if initial non-invasive attempts to restore reperfusion fail.

Acute myocardial infarctions, however, must be diagnosed and confirmed before the start of thrombolytic therapy. Therefore, we tested the sensitivity of myoglobin, creatine kinase, and creatine kinase MB in blood samples obtained before thrombolytic therapy. Neither myoglobin nor creatine kinase and creatine kinase MB were clinically efficient for diagnosing myocardial infarction within the first two hours after the onset of chest pain $(0.23 v 0.13$ and 0.05). Myoglobin was significantly more sensitive than creatine kinase $\mathrm{MB}$ activity during the 0-6 hour period and significantly more sensitive than creatine kinase activity during the 2-4 hour period after the onset of chest pain $(0.82 v 0.29)$. Myoglobin was increased in almost all patients who were admitted between the fourth and sixth hour after the onset of symptoms. The difference between the sensitivities of myoglobin and creatine kinase $(0.92$ $v 0.75)$ were not statistically significant probably because few patients $(n=12)$ were admitted during this interval. Myoglobin was significantly more sensitive in patients with myocardial infarction who presented with a non-diagnostic electrocardiogram on admission. This result accords with a recent report in which myoglobin was shown to be a strong independent predictor of myocardial infarction in patients with symptoms of short duration and to provide additional diagnostic information in patients without ST elevation. ${ }^{27}$

As a test to confirm the diagnosis of myocardial infarction myoglobin has two limitations. First, myoglobin shows earlier peak and return to normal values than creatine kinase and creatine kinase $M B$ after myocardial infarction. Myoglobin must be measured soon after a heart attack. If hospital admission is delayed for 1224 hours or more myoglobin concentrations will have fallen sharply and may be within the reference range again. Thus if chest pain is recurrent the myoglobin concentration may be 
a better indicator of further tissue necrosis than creatine kinase and creatine kinase $\mathrm{MB}$. Second, myoglobin lacks specificity for cardiac muscle. When the test result is positive the clinical setting must be taken into account. In the absence of concomitant damage to skeletal muscle or severely impaired renal function a positive myoglobin test result predicts myocardial infarction with a very high probability. The specificities of myoglobin and creatine kinase activity in patients with chest pain were surprisingly high because we studied patients in the emergency room after injured patients had preselected for treatment elsewhere. The specificity of myoglobin and creatine kinase activity in the detection of myocardial infarction will be lower in a population that includes cases of acute or chronic skeletal muscle damage caused by injury, burns, surgery, etc. A negative myoglobin assay result in the 4-12 h period after the onset of the infarct related symptoms on the other hand allows acute myocardial infarction to be ruled out within a few minutes with a very high probability.

Finally, immunoturbidimetric myoglobin measurments do contribute to the diagnosis of acute myocardial infarction in the subgroup of infarct patients presenting early with nondiagnostic electrocardiograms.

We thank Dr Gilbert Reibnegger for statistical advice and Dr Martin Hönlinger for providing plasma samples from blood donors.

1 Lee ThH, Rouan GW, Weisberg MC, et al. and the chest pain study group. Sensitivity of routine clinical criteria for diagnosing myocardial infarction within 24 hours of diagnosing myocardial infarction within 24 hou

2 Short $D$. The earliest electrocardiographic evidence of myocardial infarction. Br Heart J 1970;32:6-15.

3 Behar S, Schor S, Kavir I, Barrel V, Modan B. Evaluation of electrocardiogram in emergency room as a decision making tool. Chest 1977;71:486-91. 4 McGuiness JB, Begg TB, Semple T. First electrocar-
diogram in recent myocardial infarction. $\mathrm{Br} \mathrm{Med} J$ 1976;ii:449-51.

5 TIMI study group. The thrombolysis in acute myocardia infarction (TIMI) trial. N Engl J Med 1985;312:932-6.

6 Lee ThH, Goldman L. Serum enzyme assays in the diagnosis of acute myocardial infarction. Recommendation based on a quantitative analysis. Ann Intern Med 1986;105:221-33.

7 Drexel H, Dworzak E, Kirchmair W, Milz MM, Puschendorf B, Dienstl F. Myoglobinemia in the early phase of acute myocardial infarction. Am Heart J1983;105:642-51.
8 McComb JM, McMaster EA, MacKenzie G, Adgey AAJ. Myoglobin and creatine kinase in acute myocardial infarction. Br Heart J 1984;51:189-94.

9 Isakov A, Shapira I, Burke M, Almog Ch. Serum myoglobin levels in patients with ischemic myocardial insult. Arch Intern Med 1988;148:1762-5.

10 Gibler WB, Gibler CD, Weinshenker E, et al. Myoglobin as an early indicator of acute myocardial infarction. Ann Emerg Med 1987;16:851-6.

11 Hangaard J, Rasmussen O, Norregaard-Hansen K, Jorgensen N, Simonsen EE, Norgaard-Pedersen B. Early diagnosis of acute myocardial infarction with a rapid latex agglutination test for semi-quantitative estimation of serum myoglobin. Acta Med Scand 1987;221:343-8.

12 Ellis AK, Little T, Tasud ARZ, Liberman HA Morris DC Klocke FJ. Early non-invasive detection of successful reperfusion in patients with acute myocardial infarction. Circulation 1988;78:1352-7.

13 Katus HA, Diederich KW, Scheffold T, Uellner $M$ Schwarz F, Kübler W. Non-invasive assessment of infarct reperfusion: the predictive power of the time to peak value of myoglobin, CKMB and CK in serum. Eur Heart 1988;9:619-24.

14 World Health Organisation. WHO criteria for the diagnosis of acute myocardial infarction. Proposal for the multinational monitoring of trends and determinants in cardiovascular disease. Geneva: Cardiovascular Diseases Unit of WHO, 1981.

15 Rogers WJ, Baim DS, Gore JM, et al for the TIMI II-A Investigators. Comparison of immediate invasive, delayed invasive, and conservative strategies after tissue-type plasminogen activator: Results of the Thrombolysis in Myocardial Infarction (TIMI) Phase II-A Trial. Circulation 1990;81:1457-76.

16 Lewis BS, Ganz W, Laramee P, et al. Usefulness of a rapid initial increase in plasma creatine kinase activity as a marker of reperfusion during thrombolytic therapy for acte

17 Shell W, Mickle DK, Swan HJC. Effects of nonsurgical myocardial reperfusion on plasma creatine kinase kinetics in man. Am Heart J 1983;106:665-9.

18 Kwong TC, Fitzpatrick PG, Rothbard RL. Activities of some enzymes in serum after therapy with intracoronary streptokinase in acute myocardial infarction. Clin Chem 1984;30:731-4.

19 Ellis AK, Little T, Masud ARZ, Klocke FJ. Patterns of myoglobin release after reperfusion of injured myocardium. Circulation 1985;72:639-47.

20 Vatner SF, Baig H, Manders WT, Maroko PR. Effects of coronary reperfusion on myocardial infarct size calculated from creatine kinase. J Clin Invest 1978;61:1048-56.

21 Tuengler P, Metzmann E, Pauly HE, Becker W. New immunodiagnostic systems. Behring Inst Mitt 1988;82. 282-308.

22 Metzmann E. Protein quantitation on both branches of the Heidelberger curve by monitoring the kinetic of immunoprecipitation. Behring Inst Mitt 1985;78:167-75.

23 Gardner MJ, Altman DG. Confidence intervals rather than p values: estimation rather than hypothesis testing. $B M J$ 1986;292:746-50.

24 Albert A. On the use of likelihood ratios in clinical chemistry Clin Chem 1982;28:1113-9.

25 Gerhardt W, Keller H. Evaluation of test data from clinical studies. Scand J Clin Lab Invest 1986;(suppl 181);46: 48-64.

26 Youden WJ. Index for rating diagnostic tests. Cancer 1950; 3:32-5.

27 Ohman EM, Casey C, Bengston JR, Pryor D, Tormey W, Horgan JH. Early detection of acute myocardial infarc-
tion: additional diagnostic information from serum concentrations of myoglobin in patients without ST elevationBr Heart J 1990;63:335-8. 